\title{
A compact synchrotron radiation source driven by a laser-plasma wakefield accelerator
}

\author{
H.-P. SCHLENVOIGT ${ }^{1}$, K. HAUPT ${ }^{1}$, A. DEBUS ${ }^{1}$, F. BUDDE ${ }^{1}$, 0. JÄCKEL ${ }^{1}$, S. PFOTENHAUER ${ }^{1}$, \\ H. SCHWOERER ${ }^{1,2}$, E. ROHWER ${ }^{2}$, J. G. GALLACHER ${ }^{3}$, E. BRUNETTI ${ }^{3}$, R. P. SHANKS ${ }^{3}$, S. M. WIGGINS ${ }^{3}$ \\ AND D. A. JAROSZYNSKI ${ }^{*}$
}

\author{
${ }^{1}$ Institut für Optik und Quantenelektronik, Friedrich-Schiller-Universität, 07743 Jena, Germany \\ ${ }^{2}$ Laser Research Institute, University of Stellenbosch, 7602 Matieland, South Africa \\ ${ }^{3}$ Department of Physics, Scottish Universities Physics Alliance, University of Strathclyde, Glasgow G4 ONG, UK \\ *e-mail: d.a.jaroszynski@strath.ac.uk
}

Ultrashort light pulses are powerful tools for time-resolved studies of molecular and atomic dynamics ${ }^{1}$. They arise in the visible and infrared range from femtosecond lasers ${ }^{2}$, and at shorter wavelengths, in the ultraviolet and $\mathrm{X}$-ray range, from synchrotron sources ${ }^{3}$ and free-electron lasers ${ }^{4}$. Recent progress in laser wakefield accelerators has resulted in electron beams with energies from tens of mega-electron volts (refs 5-7) to more than $1 \mathrm{GeV}$ within a few centimetres ${ }^{8}$, with pulse durations predicted to be several femtoseconds ${ }^{9}$. The enormous progress in improving beam quality and stability ${ }^{5-8,10}$ makes them serious candidates for driving the next generation of ultracompact light sources ${ }^{11}$. Here, we demonstrate the first successful combination of a laser-plasma wakefield accelerator, producing 55-75 MeV electron bunches, with an undulator to generate visible synchrotron radiation. By demonstrating the wavelength scaling with energy, and narrow-bandwidth spectra, we show the potential for ultracompact and versatile laser-based radiation sources from the infrared to $\mathrm{X}$-ray energies.

Synchrotron light sources ${ }^{3}$ attract a large user community because they are extremely reliable sources of short radiation pulses that are tunable up to kilo-electron-volt photon energies, which make them useful for structural analysis of matter ${ }^{12}$. Furthermore, the development of free-electron lasers (FELs) ${ }^{4}$ has led to an enormous increase of brilliance and coherence. However, accelerator technology currently limits the pulse duration of synchrotron sources to just under a picosecond and demands large and expensive facilities because the accelerating field gradients of conventional accelerators are restricted to $20-100 \mathrm{MV} \mathrm{m}^{-1}$ by radiofrequency-cavity electrical breakdown.

In contrast, a plasma, which is already fully broken down, can sustain electric fields that are 3-4 orders of magnitude higher, exceeding $100 \mathrm{GV} \mathrm{m}^{-1}$. In the late $1970 \mathrm{~s}$, Tajima and Dawson ${ }^{13}$ proposed harnessing the large ponderomotive forces-arising from the light pressure exerted by intense laser fields-to excite plasma waves that travel close to the speed of light and trail behind the laser pulse. The electrostatic forces of such a plasma wake (as found behind a boat) rapidly accelerate particles to very high energies. Energy is gained analogously to a surfer acquiring momentum from a water wave. The necessary light intensities of more than $10^{18} \mathrm{~W} \mathrm{~cm}^{-2}$ can be obtained from femtosecond laser pulses, which are amplified using the chirped pulse amplification technique $^{14}$ to peak powers exceeding tens of terawatts and peak intensities at the laser beam focus of more than $10^{20} \mathrm{~W} \mathrm{~cm}^{-2}$. The availability of reliable high-power lasers has led to enormous progress in the development of laser-plasma wakefield accelerators. Insights from three-dimensional particle-in-cell simulations ${ }^{9}$ have increased our understanding of the conditions necessary to controllably accelerate monoenergetic particles that are injected directly from the background plasma. This led, in 2004, to the first experimental demonstration of controlled plasma wakefield acceleration over several millimetres ${ }^{5-7}$. More recently, through the development of plasma channel waveguides to preserve the laser intensity over several centimetres, bunches of electrons have been reproducibly accelerated to $0.5-1 \mathrm{GeV}$, with a few per cent bandwidth measured energy spread and within a wellcollimated beam containing tens to hundreds of picocoulombs of charge ${ }^{8}$. Further control of the reproducibility and energy has been demonstrated using a counter-propagating laser pulse ${ }^{10}$. As the scale length of the wakefield accelerating potential is determined primarily by the period of the plasma oscillation, which is of the order of the laser pulse duration (30-100 fs), the electron bunches are inherently ultrashort, with durations predicted to be $10 \mathrm{fs}$ or less ${ }^{9}$.

The experiment to demonstrate the production of undulator synchrotron radiation with laser-accelerated electrons has been carried out using the high-intensity titanium:sapphire laser JETI at Jena ${ }^{15}$. The set-up (see Fig. 1) consists of a laser wakefield accelerator as a source of relativistic electrons, driven by an $80 \mathrm{fs}$ laser pulse with an intensity of $5 \times 10^{18} \mathrm{~W} \mathrm{~cm}^{-2}$ (ref. 16). The electrons traverse a 50 period, $1-\mathrm{m}$-long undulator with a deflection parameter $K=0.6$, and are then analysed by a calibrated electron energy spectrometer. Undulator radiation generated by electron bunches in the range of $55-75 \mathrm{MeV}$ is collected by a lens and focused into a Czerny-Turner spectrograph equipped with a highsensitivity CCD (charge-coupled device) camera. It is important to note that the electron and the undulator radiation spectra are 


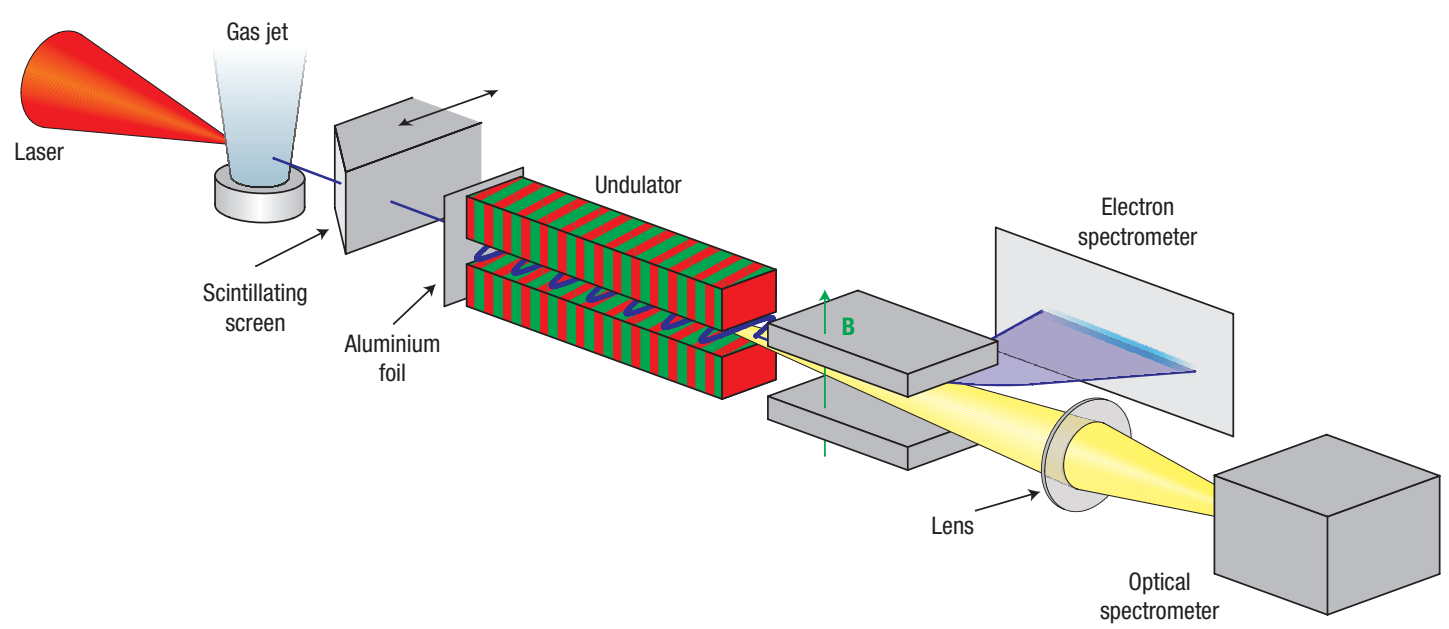

Figure 1 Set-up of the experiment. The laser pulse is focused by an off-axis parabolic mirror into a supersonic helium gas jet where it accelerates electrons (blue line) to several tens of mega-electron volt energy. The electron beam profile may be monitored by a removable scintillating screen. The electrons propagate through an undulator, producing synchrotron radiation, and into a magnetic electron spectrometer. Radiation is collected by a lens and analysed in an optical spectrometer. The spectrometer is protected against direct laser and plasma exposure by a thin aluminium foil in front of the undulator.

simultaneously recorded for each individual shot, as shown in the Methods section.

Relativistic electrons undergo transverse oscillations as they pass through the undulator under the action of the Lorentz force of the periodic magnetic field and emit polarized radiation. The wavelength $\lambda$ of the emitted light is mainly given by the undulator period $\lambda_{\mathrm{u}}$ and the electron energy $E_{\mathrm{e}}=\gamma m_{0} c^{2}$, where $\gamma$ is the relativistic Lorentz factor, $m_{0}$ is the electron rest mass and $c$ is the speed of light. The small reduction in the longitudinal velocity due to periodic deflection results in a slight decrease in the frequency of the radiation field by a factor of $\left(1+K^{2} / 2\right)$. The wavelength of the emitted radiation also depends on the angle of emission, $\vartheta$, with respect to the optical axis and is peaked in the forward direction. This is given by $\lambda=\left(\lambda_{\mathrm{u}} / 2 h \gamma^{2}\right)\left(1+\left(K^{2} / 2\right)+\gamma^{2} \vartheta^{2}\right)$, where $h$ is the harmonic order. For electron energies of $55-75 \mathrm{MeV}$, the wavelength emitted by our undulator is in the visible spectral range.

Figure 2 shows a measured electron spectrum (inset) and the corresponding undulator radiation spectrum (black squares) for the same laser shot. The electron spectrum is peaked at $64 \mathrm{MeV}$, has a width of $3.4 \mathrm{MeV}$ (full-width at half-maximum, FWHM) and contains a charge of $28 \mathrm{pC}$. As the electron beam is not focused into the undulator, the relevant Twiss beam parameters at the centre of the undulator are $\beta=100 \mathrm{~m}$ and $\alpha=-115$. The normalized emittance of the beam is taken as $\varepsilon_{\mathrm{n}} \approx 1.3 \pi \mathrm{mm}$ mrad, which has been estimated from the source size, $\sigma_{\text {r.m.s. }}=3 \mu \mathrm{m}$ derived from simulations and the beam divergence $\sigma_{\text {r.m.s. }}^{\prime}=1.6 \mathrm{mrad}$ determined from the beam spot size. This value of emittance is consistent with simulations ${ }^{9}$. The measured undulator radiation spectrum, shown in Fig. 2, is peaked at $740 \mathrm{~nm}$, has a bandwidth of $55 \mathrm{~nm}$ and contains 284,000 photons. We also show a similar peak (red circles) at $900 \mathrm{~nm}$ produced by a $58 \mathrm{MeV}, 14 \mathrm{pC}, \sigma_{\gamma} / \gamma \approx 5 \%$, bunch in another shot (not shown). The expected undulator radiation spectrum has been calculated for the measured electron spectra, taking into account the $1 \mathrm{MeV}$ resolution of the spectrometer, by integrating the Liénard-Wiechert potentials ${ }^{17,18}$ for a collection angle of 2 mrad. The calculated undulator spectra indicated by the solid lines in Fig. 2 are in excellent quantitative agreement with the measured photon spectra peak positions, spectral widths and photon numbers.

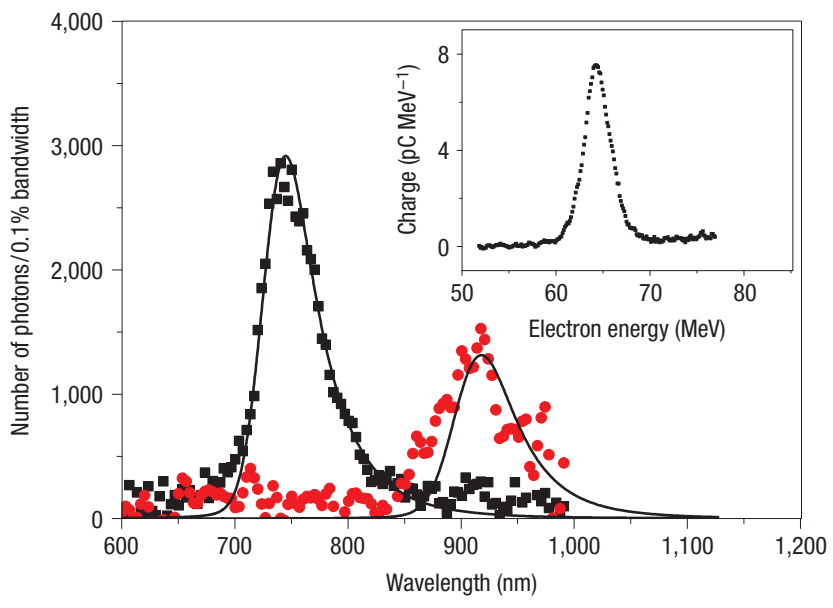

Figure 2 Undulator radiation spectrum and corresponding electron spectrum. The measured electron spectrum (inset) shows a distinct maximum at $64 \mathrm{MeV}$ with a width of $3.4 \mathrm{MeV}$ (FWHM), and a total charge of $28 \mathrm{pC}$. The corresponding undulator radiation spectrum (black squares) is simultaneously recorded. The peak centred at $740 \mathrm{~nm}$ contains 284,000 photons. The red circles show an undulator radiation spectrum from a different shot, produced by a $58 \mathrm{MeV}, 14 \mathrm{pC}$ electron bunch. The simulations of the undulator spectra (solid lines) take into account the measured electron spectrum, the undulator parameters and the optical imaging system, and compare well with the measured signals.

The measured optical spectrum provides a valuable method for estimating the beam quality and sets an upper limit on the energy spread and emittance. The total relative spectral width must include contributions from the natural spectral width $\delta \lambda / \lambda=1 / N_{\mathrm{u}}$, which is $2 \%$ for our undulator. The measured spectral width imposes an upper limit on the combined angular spread, $\vartheta^{2} \gamma^{2}$, and energy spread, $2 \sigma_{\gamma} / \gamma$, because $(\delta \lambda / \lambda)_{\text {measured }}^{2}=\left(2 \sigma_{\gamma} / \gamma\right)^{2}+\left(\vartheta^{2} \gamma^{2}\right)^{2}+1 / N_{\mathrm{u}}^{2}$. This places an upper limit on the emittance of $\varepsilon_{\mathrm{n}} \approx 1 \pi \mathrm{mm}$ mrad and electron beam 


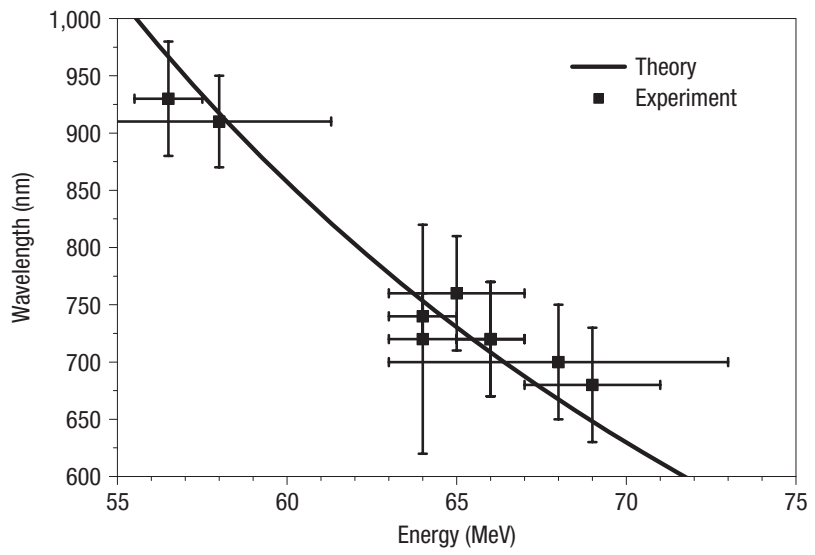

Figure 3 Correlation between electron energy and undulator radiation. The squares represent the centre of the measured undulator radiation wavelength as a function of the electron energy for various laser shots. The data are taken from shots producing narrow-band electron spectra within the range $55-75 \mathrm{MeV}$. The bars represent the spectral widths, of both electron (horizontal) and photon (vertical) signals. Note: they are not error bars. The data are compatible with the theoretical dependence of undulator wavelength on electron energy, which is shown as a solid line.

relative energy spread, $\sigma_{\gamma} / \gamma \approx 1 \%$, which is less than the measured energy spread $\sigma_{\gamma} / \gamma \approx 2.2 \%$ and consistent with the $1.6 \%$ resolution of the electron spectrometer at $65 \mathrm{MeV}$.

The very short electron bunch estimated to be $10 \mathrm{fs}$ results in a very high peak brilliance (photon flux per unit solid angle per unit area at the source ${ }^{3}$ ) of the order of $6.5 \times 10^{16}$ photons $/ \mathrm{s} / \mathrm{mrad}^{2} / \mathrm{mm}^{2} / 0.1 \%$ bandwidth. If we assume the 200 period, $K=1,3$-m-long undulator ${ }^{11}$ with the $1 \mathrm{GeV}$ beams that have recently been produced ${ }^{8}$, we obtain 220,000 photons in the first harmonic with an energy of $420 \mathrm{eV}$ in $0.1 \mathrm{mrad}$ with a peak brilliance $2.5 \times 10^{24}$ photons $/ \mathrm{s} / \mathrm{mrad}^{2} / \mathrm{mm}^{2} / 0.1 \%$ bandwidth for a $28 \mathrm{pC}, 10$-fs-duration electron bunch (with $\varepsilon_{\mathrm{n}} \approx 1 \pi \mathrm{mm}$ mrad, $\sigma_{\gamma} / \gamma \approx 1 \%$ and $\left.\beta=1 \mathrm{~m}\right)$.

Figure 3 shows the measured correlation between electron energy and undulator radiation wavelength. The squares represent different shots showing a narrow electron spectrum with a peak energy in the range of $55-75 \mathrm{MeV}$, and the corresponding peak wavelengths from simultaneously measured undulator spectra. The horizontal and vertical bars represent the spectral width of electron and photon signals, respectively. The peak wavelengths correspond very well to the expected undulator wavelength (see the solid line) and residual scatter arises from the shot-to-shot pointing variations which are of the order $\sigma_{\vartheta} \approx 3 \mathrm{mrad}$. Electron bunches with energies below $55 \mathrm{MeV}$-corresponding to undulator radiation beyond the optical spectrometer's range-produced no optical signal, except for a few shots with high charge around $45 \mathrm{MeV}$ that showed a faint signal at wavelengths corresponding to second-harmonic undulator radiation.

The wavelength scaling with electron energy, absolute photon numbers and measured spectral shapes are unambiguous evidence that the observed emission is undulator radiation. Plasma radiation, a strong broadband emission independent of the electron energy, is entirely shielded by an aluminium foil (see Fig. 1). Coherent and incoherent transition radiation is produced by the short electron bunch as it crosses the boundary between the plasma and the vacuum ${ }^{19,20}$, and also as it passes through the aluminium foil. However, transition radiation is again broadband and predominantly emitted in lobes at an angle of $1 / \gamma$ from the electron beam axis ${ }^{17}$. Coherent transition radiation due to short length-scale density modulation of the electron bunch arising from the electric field of the laser pulse $\mathrm{e}^{20}$ is avoided by placing the foil $400 \mathrm{~mm}$ from the gas jet. The modulations are rapidly washed out by momentum dispersion caused by space-charge explosion of the electron bunch. Consequently, we must conclude that the observed narrow-band radiation is indeed generated by electrons emitting in the undulator.

Compact radiation sources based on wakefield accelerators should have a number of advantages over conventional sources. Owing to the $\gamma^{2}$ scaling of undulator radiation frequency, sub-100-fs, incoherent light pulses in the ultraviolet and soft $\mathrm{X}$-ray spectral range will be produced, as described here, using today's high-intensity lasers ${ }^{8}$. The generation of coherent undulator radiation is more challenging. With current wakefield accelerators this might be possible in the infrared spectral range because coherence is ensured by the short effectively pre-bunched electron beam $^{21}$. FELs operating in the ultraviolet or X-ray region rely on self-amplified spontaneous emission, which requires electron beams with extremely small emittance and long and highly engineered undulators. However, the self-amplified spontaneous emission process also depends on the current density of the electron beam, which could be very high for wakefield accelerators, exceeding tens of kiloamperes. Therefore, with future reduction in energy spread, laser-plasma accelerators may be optimal compact drivers of X-ray FELs ${ }^{11}$. Thomson backscattering off the laser pulse is an alternative method of producing incoherent X-ray pulses, but with relatively broad spectral bandwidth and low efficiencies, using current Ti:sapphire lasers ${ }^{15}$. A source of broad-bandwidth $\mathrm{X}$-ray radiation is betatron-like wiggler radiation from electrons undergoing transverse motion in the accelerating potential of the wakefield ${ }^{22,23}$. However, the advantage of using a separate undulator is relatively narrow-bandwidth radiation can be produced with high peak brilliance and the accelerator and radiator are decoupled thus allowing better control of the properties of the radiation.

Laser-based accelerators imply a large reduction in infrastructure, particularly for shielding, and thus in size and $\operatorname{cost}^{11}$. A purely laser-driven light source would inherently produce ultrashort-duration pulses and be perfect temporally synchronized with the driving laser. Time-resolved experiments require at least two pulses, one to initiate the fast process to be observed and another to probe after a well-defined delay. The pump and probe would automatically be synchronized with the electron bunch and thus the undulator radiation. However, there we are not restricted to laser pulses, and these may be converted to another form, for example, to an electron pulse or proton pulse $\mathrm{e}^{24,25}$. Thus, a laserbased accelerator facility would be very flexible.

Laser-wakefield-based facilities could form a new type of compact light source and underpin a wide range of applications such as time-resolved X-ray diffraction, photoelectron spectroscopy, ultrafast studies of material damage, plasma physics, radiography of dense materials, X-ray microscopy and X-ray imaging, and could produce a revolution in the way science is done. This experiment marks an important step towards the application of high-intensity laser accelerators in conventional accelerator science and compact light-source development.

\section{METHODS}

\section{LASER-PLASMA WAKEFIELD ACCELERATOR}

The JETI laser delivers laser pulses at $795 \mathrm{~nm}$ central wavelength with an energy of $430 \mathrm{~mJ}$ on target within a pulse duration of $85 \mathrm{fs}$. The laser pulses are focused by an F/6 $30^{\circ}$ off-axis parabolic mirror to a spot area of $95 \mu \mathrm{m}^{2}$ (FWHM of energy), yielding a peak intensity of $5 \times 10^{18} \mathrm{~W} \mathrm{~cm}^{-2}$, corresponding to 
a normalized vector potential $a_{0}=1.5$. A pulsed supersonic helium gas jet is positioned at the focal spot, generating a $2-\mathrm{mm}$-diameter supergaussian gas density distribution with a peak gas density of $2 \times 10^{19} \mathrm{~cm}^{-3}$. As $a_{0}>1$, electrons are efficiently accelerated to relativistic energies by laser-plasma wakefield acceleration. Nonlinear Thomson scattering from the plasma channel is recorded for each individual laser shot for online diagnosis of the laser-plasma interaction. Before taking undulator shots, the electron beam profile, divergence and pointing direction are observed on removable screens and optimized by varying gas density, gas jet position and direction of laser incidence. Because of small fluctuations in the gas and laser parameters, and the highly nonlinear nature of laser propagation and electron acceleration, only about 1 in 10 laser pulses produced monoenergetic electron bunches. Furthermore, the mean energy, charge and the energy spectral width and shape varied considerably from shot to shot. Recent experiments ${ }^{8,10}$ have shown that the reproducibility and stability can be increased to better than $5 \%$ from shot to shot.

\section{UNDULATOR}

After removing the scintillating screen, the electrons propagate through an undulator, placed $40 \mathrm{~cm}$ behind the gas jet. The fixed-gap undulator is constructed from permanent magnets in a hybrid structure (period $=2 \mathrm{~cm}$, $N=50$ periods, gap $=10 \mathrm{~mm}$, maximum magnetic field strength on-axis $B=330 \mathrm{mT})$. The undulator parameter, defined as $K=\left(e B \lambda_{\mathrm{u}}\right) /\left(2 \pi m_{0} c\right)$, is $K=0.6$. The first and last three periods of the undulator have been carefully adjusted for on-axis injection and on-axis exit of the electrons. The undulator is located inside the vacuum vessel. Furthermore, the magnets inside the undulator are protected from electrons propagating off-axis by a $1-\mathrm{cm}$-thick 1-cm-diameter lead aperture.

\section{ELECTRON SPECTROMETER}

The electron spectrometer (permanent magnets $200 \mathrm{~mm} \times 100 \mathrm{~mm}, 20 \mathrm{~mm}$ gap, central magnetic field strength $720 \mathrm{mT}$ ) is placed $185 \mathrm{~cm}$ behind the gas jet. A scintillating screen (Konica KR) in combination with a CCD camera is used to detect electrons in the energy range $14-85 \mathrm{MeV}$. The screen images have been calibrated against an imaging plate (Fuji BAS-MS2025) to give an absolute measure of charge per unit energy taking into account the response of the imaging plate and the scintillating screen, respectivel ${ }^{26,27}$.

\section{OPTICAL SPECTROMETER}

Undulator radiation is collected within a full solid angle of $0.3 \mathrm{msr}$ and focused into the entrance slit plane of a symmetrical $200 \mathrm{~mm}$ Czerny-Turner spectrometer. A thermoelectrically cooled CCD camera (Andor DO-420 BN) is used as a detector. The CCD chip $(1,024 \times 256$ pixels $)$ operates in a hardware binning mode, merging arrays of $8 \times 12$ pixels into superpixels. The spectral range is set to $540-990 \mathrm{~nm}$. The spectrometer and detector efficiency has been carefully calibrated using a calibrated visible-light source. The optical spectrometer is screened against direct exposure from the laser and plasma light by a $15-\mu \mathrm{m}$-thick aluminium foil placed in front of the undulator.

Received 16 August 2007; accepted 12 November 2007; published 9 December 2007.
References

1. Zewail, A. Nobel 47 Lecture (1999) 274-367 (Nobel Lectures in Chemistry 1996-2000, World Scientific, Singapore, 2003).

2. Squier, J., Salin, F., Mourou, G. \& Harter, D. 100-fs pulse generation and amplification in Ti: $\mathrm{Al}_{2} \mathrm{O}_{3}$. Opt. Lett. 16, 324-326 (1991).

3. Bilderback, D. H., Elleaume, P. \& Weckert, E. Review of third and next generation synchrotron light sources. J. Phys. B 38, S773-S797 (2005).

4. Ayvazyan, V. et al. First operation of a free-electron laser generating GW power radiation at $32 \mathrm{~nm}$ wavelength. Eur. Phys. J. D 37, 297-303 (2006).

5. Mangles, S. P. D. et al. Monoenergetic beams of relativistic electrons from intense laser plasma interactions. Nature 431, 535-538 (2004).

6. Geddes, C. G. R. et al. High quality electron beams from a laser wakefield accelerator using plasma-channel guiding. Nature 431, 538-541 (2004).

7. Faure, J. et al. A laser-plasma accelerator producing monoenergetic electron beams. Nature 431, 541-544 (2004).

8. Leemans, W. P. et al. GeV electron beams from a centimetre-scale accelerator. Nature Phys. 2, 696-699 (2006).

9. Pukhov, A. \& Meyer-ter Vehn, J. Laser wake field acceleration: The highly non-linear broken-wave regime. Appl. Phys. B 74, 355-361 (2002).

10. Faure, J. et al. Controlled injection and acceleration of electrons in plasma wakefields by colliding laser pulses. Nature 444, 737 (2006).

11. Jaroszynski, D. A. et al. Radiation sources based on laser-plasma interactions. Phil. Trans. R. Soc. A 364, 689 (2006).

12. Chapman, H. et al. Femtosecond diffractive imaging with a soft-X-ray free-electron laser. Nature Phys. 2, 839-843 (2006).

13. Tajima, T. \& Dawson, J. M. Laser electron accelerator. Phys. Rev. Lett. 43, 267 (1979).

14. Pittman, M. et al. Design and characterization of a near-diffraction-limited femtosecond 100-TW 10-Hz high-intensity laser system. Appl. Phys. B 74, 529-535 (2002).

15. Schwoerer, H. et al. Thomson-backscattered $\mathrm{x}$ rays from laser-accelerated electrons. Phys. Rev. Lett. 96, 014802 (2006).

16. Hidding, B. et al. Generation of quasimonoenergetic electron bunches with 80 -fs laser pulses. Phys. Rev. Lett. 96, 105004 (2006).

17. Jackson, J. D. Classical Electrodynamics (Wiley, New York, 1975).

18. Tanaka, T. \& Kitamura, H. SPECTRA: A synchrotron radiation calculation code. J. Synchrotron Radiat. 8, 1221 (2001).

19. van Tilborg, J. et al. Temporal characterization of femtosecond laser-plasma-accelerated electron bunches using terahertz radiation. Phys. Rev. Lett. 96, 014801 (2006).

20. Glinec, Y. et al. Observation of fine structures in laser-driven electron beams using coherent transition radiation. Phys. Rev. Lett. 98, 194801 (2007).

21. Jaroszynski, D. A. et al. Superradiance in a short-pulse free-electron-laser oscillator. Phys. Rev. Lett. 78, 1699-1702 (1997)

22. Rousse, A. et al. Production of a keV X-ray beam from synchrotron radiation in relativistic laser-plasma interaction. Phys. Rev. Lett. 93, 135005 (2004).

23. Phuoc, K. T. et al. Imaging electron trajectories in a laser-wakefield cavity using betatron X-ray radiation. Phys. Rev. Lett. 97, 225002 (2006).

24. Schwoerer, H. et al. Laser-plasma acceleration of quasi-monoenergetic protons from microstructured targets. Nature 439, 445-448 (2006).

25. Hegelich, M. et al. Laser acceleration of quasi-monoenergetic $\mathrm{MeV}$ ion beams. Nature 439, 441-444 (2006).

26. Glinec, Y. et al. Absolute calibration for a broad range single shot electron spectrometer. Rev. Sci. Instrum. 77, 103301 (2006).

27. Tanaka, K. A. et al. Calibration of imaging plate for high energy electron spectrometer. Rev. Sci. Instrum. 76, 013507 (2005).

Acknowledgements

This work was supported by the Deutsche Forschungsgemeinschaft under contract TR18. Financial support by the Access to Research Infrastructures activity in the Sixth Framework Programme of the EU (contract RII3-CT-2003-506350, Laserlab Europe) for conducting the research is gratefully the EU (contract RII3-CT-2003-506350, Laserlab Europe) for conducting the research is gratefully
acknowledged. We thank B. Beleites, F. Ronneberger and W. Ziegler for their technical support. S.M.W. acknowledges the support of the Department of Physics, Lancaster University, and the Cockcroft Institute, Daresbury Laboratory, Daresbury, UK. The UK team also acknowledges the support of the 105 Research Councils UK, EPSRC and the EU EuroLEAP NEST contract no. 028514. Correspondence and requests for materials should be addressed to D.A.J.

Reprints and permission information is available online at http://npg.nature.com/reprintsandpermissions/ 